\title{
Enhanced Recovery After Surgery (ERAS)
}

\author{
Gregory L. Bryson, MD
}

Received: 6 November 2014/ Accepted: 11 November 2014/Published online: 9 December 2014

(C) Canadian Anesthesiologists' Society 2014

\section{Multiple Choice Questions}

The multiple choice questions which appear below relate to the series of special review articles in the February 2015 issue of the Journal. For each question, select the one best response. The correct responses appear online as Electronic Supplemental Material.

Physiologic considerations of Enhanced Recovery After Surgery (ERAS) programs: implications of the stress response

1. Which of the following is not a component of Enhanced Recovery After Surgery?
a. Preoperative carbohydrate drink
b. Laparoscopy
c. Normothermia
d. Chemotherapy

2. Which statement concerning insulin resistance is correct?

a. It is associated with hyperglycemia and protein breakdown

b. It is responsible for hyperkalemia during surgery

c. It is measured in the blood

d. It impacts patients' ability to increase glucose and glycogen

Electronic supplementary material The online version of this article (doi:10.1007/s12630-014-0277-8) contains supplementary material, which is available to authorized users.

G. L. Bryson, MD

University of Ottawa, Ottawa, Ontario, Canada

e-mail: glbryson@ottawahospital.on.ca
3. Which statement is incorrect?
a. Hyperglycemia is associated with postoperative complications
b. Insulin resistance is associated with low levels of insulin in the blood
c. HbA1c $>6.5 \mathrm{mmol} \cdot \mathrm{L}^{-1}$ indicates diabetes
d. Epidural anesthesia and analgesia completely inhibits the stress response

What outcomes are important in the assessment of Enhanced Recovery After Surgery (ERAS) pathways?

4. The primary outcome most commonly reported in studies comparing an enhanced recovery program with conventional care after surgery is:
a. Complications
b. Length of stay
c. Mobilization
d. Time to readiness for discharge

5. Patients equate recovery from surgery with:
a. Absence of complications
b. Adequate pain control
c. Return to normal activities
d. Discharge from hospital

6. In quantitative meta-analyses, benefits of enhanced recovery after surgery programs include:
a. Higher patient satisfaction
b. Less pain
c. Better quality of life
d. Shorter hospital stay 
Cardiopulmonary exercise testing, prehabilitation, and Enhanced Recovery After Surgery (ERAS)

7. All of the following cardiopulmonary exercise testing (CPET) derived variables have been shown to be associated with outcome (morbidity or mortality) following elective major surgery except:
a. Ventilatory equivalents for carbon dioxide (VE/ $\mathrm{VCO}_{2}$ )
b. Peak oxygen consumption $\left(\mathrm{mL} \cdot \mathrm{kg}^{-1} \cdot \mathrm{min}^{-1}\right)$
c. Oxygen consumption at anaerobic threshold $\left(\mathrm{mL} \cdot \mathrm{kg}^{-1} \cdot \mathrm{min}^{-1}\right)$
d. Ventilatory equivalents for oxygen $\left(\mathrm{VE} / \mathrm{VO}_{2}\right)$
e. The oxygen pulse at $\dot{V} O_{2}$ peak TRUE

8. Which of the following is not true of CPET and exercise training perioperatively?
a. Neoadjuvant chemotherapy is associated with a reduction in exercise capacity
b. Preoperative exercise training improves exercise capacity
c. Preoperative exercise training may improve clinical outcomes after surgery
d. Neoadjuvant chemotherapy improves $\dot{V} \mathrm{O}_{2}$ peak
e. Post chemotherapy training can improve exercise capacity

9. All but one of the following clinical areas has currently available clinical trial data:

a. The impact of neoadjuvant chemotherapy on physical fitness (defined by CPET)

b. The impact of neoadjuvant chemoradiotherapy on physical fitness (defined by CPET)

c. The impact of immunotherapy on physical fitness (defined by CPET)

d. The impact of physical fitness (defined by CPET) on surgical outcome

e. The impact of using CPET to guide choice of postoperative care environment (but this is from a study with historical controls; there is no randomized data available)

\section{Frailty and perioperative outcomes: a narrative review}

10. Frailty is:
a. Multifactorial
b. Synonymous with aging
c. Seen frequently in the older adult population
d. All of the above
e. A and C only

11. Which of the following is true:

a. Frailty is a distinct pathophysiological entity

b. The modified frailty index is the best tool for scoring frailty in surgical patients

c. The bulk of frailty assessments focus on 5 core characteristics from the Fried Criteria that include cognitive dysfunction, weakness, low physical activity, exhaustion and shrinking

d. The Fried frailty criteria has demonstrated a strong association with adverse outcomes in frail patients including an increased risk for falls, hospitalization, disability and death

e. Frailty is only seen in patients who are $65 \mathrm{yr}$ of age and older

12. Which of the following best represents "frailty":
a. Aging $\rightarrow$ Disease $\rightarrow$ Disability $\rightarrow$ Adverse Outcome
b. Biological reserve $\rightarrow$ Inability to cope with stressors $\rightarrow$ Vulnerability $\rightarrow$ Adverse Outcomes
c. Sarcopenia $=$ Disability $=$ Frailty
d. Aging $=$ Frailty
e. None of the above

Fluid management and goal-directed therapy as an adjunct to Enhanced Recovery After Surgery (ERAS)

13. Concerning preoperative management of Enhanced Recovery After Surgery (ERAS) patients

a. All patients should be nil by mouth from midnight to reduce the risk of aspiration

b. Patients should receive $1 \mathrm{~L}$ of intravenous fluid before induction to replace the deficit from overnight fasting

c. Mechanical bowel preparation decreases the fecal load in the bowel, thereby decreasing the risk of wound infections and anastomotic breakdown

d. A preoperative carbohydrate drink can reduce thirst, hunger, and postoperative insulin resistance

14. Goal-directed fluid therapy

a. Utilizes high volumes of crystalloid to optimize stroke volume and maintain urine output

b. Has been shown to be beneficial in all studies within ERAS programs

c. Has been shown to cause harm to patients within an ERAS program

d. Should ultimately be specific to the surgeon, patient, and institute 
15. With regard to dynamic variables of fluid responsiveness such as stroke volume variation

a. They are most useful to detect volume responsiveness in laparoscopic surgery in the steep Trendelenburg position

b. They enable episodes of hypovolemia to be detected before there is any change in heart rate or blood pressure

c. If a patient is shown to be volume responsive, they always need volume

d. They can be used preoperatively in awake patients to optimize hemodynamics

16. Low perioperative urine output

a. Is a sign of developing renal failure and is best treated with aggressive fluid boluses

b. Is strongly predictive of a rise in postoperative creatinine

c. Should be tolerated in the absence of other concerns, as it is not a reliable indicator of volume status

d. Is a reliable indicator of intravascular hypovolemia

\section{Inter-device differences in monitoring for goal-directed fluid therapy}

17. You are trying to measure the velocity of blood flow through the aortic valve using Doppler. Assuming that the actual angle of incidence $(\theta)$ during the measurement is about $30^{\circ}$ and no adjustment is made, which of the following statements is most accurate?
a. The Doppler device will accurately estimate the velocity
b. The Doppler device will underestimate the velocity
c. The Doppler device will overestimate the velocity
d. Cannot be calculated

18. Which of the formulas below best represents arterial pulse pressure variation (PPV) according to the Figure (available as Electronic Supplemental Material) below?
a. $\frac{(\mathrm{A}-\mathrm{B})-(\mathrm{C}-\mathrm{D}) .}{[(\mathrm{A}-\mathrm{B})+(\mathrm{C}-\mathrm{D})] / 2}$
b. B-D
c. $(\mathrm{A} / \mathrm{B}) \times(\mathrm{C} / \mathrm{D})$
d. A-C

19. Cardiac output measurement using esophageal Doppler monitoring (EDM) is subject to which of the following sources of error?
a. Unmeasured flow to the upper extremities and head
b. Estimation of aortic cross-sectional area
c. Estimation of angle of incidence $\theta$
d. Changes in afterload
e. All of the above

20. The passive leg raising maneuver is a useful bedside examination technique to evaluate which of the following parameters?
a. Central venous pressure
b. Systemic vascular resistance
c. Pulse pressure variation
d. Response to fluid administration

\section{Perioperative catabolism}

21. Which statement about the catabolic response to surgery is true?

a. In non-diabetic patients undergoing abdominal surgery, blood glucose levels typically increase to $12-15 \mathrm{mmol} \cdot \mathrm{L}^{-1}$

b. The perioperative administration of corticosteroids has no effect on blood glucose concentration

c. The magnitude of insulin resistance is most pronounced one day after surgery and lasts for about three weeks after abdominal surgery

d. Protein loss in type 2 diabetic patients after colorectal cancer surgery has been shown to be $5 \%$ greater than in non-diabetics

22. Which statements about insulin are true?

a. Using so-called insulin sliding scales, intraoperative normoglycemia can be maintained even during open heart surgery

b. The majority of randomized controlled trials on glycemic control achieved normoglycemia in the therapy group

c. Insulin has anti-inflammatory, cardioprotective, and inotropic properties at supraphysiological doses

d. Most current guidelines recommend treatment of glucose levels $>10 \mathrm{mmol} \cdot \mathrm{L}^{-1}$ 
23. Which of the following statements is true?

a. Perioperative nutrition causes less protein anabolism in malnourished patients than in well-nourished patients

b. Energy expenditure, as measured by indirect calorimetry, typically correlates well with calculated values using the Harris-Benedict equation

c. In large randomized clinical trials on glucose control in critically ill patients, such as the Leuven II and the VISEP study, severe hypoglycemia was a rare event $(<5 \%)$

d. A large variation of blood glucose may be harmful, independent of the absolute mean glucose level

Prevention of venous thromboembolism in the Enhanced Recovery After Surgery (ERAS) setting: an evidence-based review

24. A 76-yr-old woman with body mass index 26 is scheduled to undergo an urgent total abdominal hysterectomy with bilateral salpingo-oophorectomy for suspected ovarian cancer. The most appropriate deep vein thrombosis (DVT) prophylaxis regimen is:
a. Dalteparin $200 \mathrm{IU} \cdot \mathrm{kg}^{-1} s c$ once daily for the duration of her hospitalization
b. Enoxaparin $40 \mathrm{mg} \mathrm{sc}$ once daily for 28 days
c. Acetylsalicylic acid $160 \mathrm{mg}$ po once daily for the duration of her hospitalization
d. Fondaparinux $2.5 \mathrm{mg} \mathrm{sc}$ once daily for 28 days

25. A 54-yr-old man with a bare-metal stent inserted into his left anterior descending coronary artery six months ago following an acute coronary syndrome requires an emergent Hartmann procedure for sigmoid diverticulitis with abscess. He is not obese. With respect to perioperative arterial and venous thromboprophylaxis, he should receive:
a. Acetylsalicylic acid $81 \mathrm{mg}$ once daily indefinitely plus dalteparin 5,000 IU sc once daily for the duration of his hospitalization
b. Dalteparin 5,000 IU sc once daily for the duration of his hospitalization
c. Acetylsalicylic acid $160 \mathrm{mg}$ daily
d. Apixaban $2.5 \mathrm{mg}$ twice daily

26. Higher doses of DVT prophylaxis should be considered for obese patients.
a. True
b. False

27. The direct-acting oral anticoagulants are proven to be safe and effective for DVT prophylaxis in patients undergoing abdominal/pelvic surgery.
a. True
b. False

Optimizing pain management to facilitate Enhanced Recovery After Surgery pathways

28. Almost $90 \%$ of patients experience some degree of pain after surgery. How many patients have moderateto-extreme pain in the immediate postoperative period?
a. $25 \%$
b. $50 \%$
c. $75 \%$
d. $100 \%$

29. Which of the following analgesic modalities has the strongest evidence for reducing time to return of gastrointestinal function (passing flatus or bowel movement)?
a. Surgical site infiltration
b. Epidural analgesia
c. Intravenous acetaminophen
d. NSAIDs
e. Tramadol
f. NMDA receptor antagonists

30. Which of the following modalities is associated with the shortest length of hospital stay?
a. Neuraxial or regional blocks
b. Systemic opioids
c. Systemic non-opioid analgesia
d. Non-pharmacological methods 
Health economics in Enhanced Recovery After Surgery programs

31. A 60-yr-old male underwent open right hemicolectomy. His perioperative care was managed according to the institute's Enhanced Recovery After Surgery (ERAS) program in colorectal surgery. He required 2 units of blood on day 2 as his hemoglobin concentration $(\mathrm{Hb})$ was 76 $\mathrm{g} \cdot \mathrm{L}^{-1}$ and he was symptomatic. Thereafter, his postoperative recovery was uneventful, and he was discharged from hospital on day 5 .

In this scenario, the following are all examples of costs incurred from a societal perspective, EXCEPT:

a. The patient continued to feel tired for two weeks after his operation and was unable to return to work as an accounts manager until the third week

b. The patient encountered some wound problems ten days after the operation with the distal end of the wound dehiscing. His family doctor organized daily visits from district nurses until the wound had healed

c. The patient received two units of blood while in hospital

d. The patient's wife, who works as a sales manager, took two weeks off work to care for her husband

e. The patient's wound problems initially caused him anxiety, and subsequently, his wounds were slow to heal. Moreover, his wife noticed that he was not his usual self, reporting his mood to be low

32. The studies included in this review have reported cost data mostly from the payer's perspective (direct inhospital costs). From the following, choose the list that reports costs only from a payer's perspective:

a. Preoperative imaging, preoperative assessment, attendance at a group education session, employment of an ERAS nurse specialist, operative time

b. Discharge medications, readmission, attendance at the Accident and Emergency Department, follow-up phone call, postop clinic appointment

c. District nurse follow-up, outpatient physiotherapy, readmission, postoperative $x$-rays, inability to participate in usual activities

d. Operation, anesthetic, surgeon time, perioperative intravenous fluids, convalescence at home

e. Time off work, return to theatre, caregiver burden, postoperative analgesia
33. Quality of life data should be collected when measuring the indirect costs incurred through management of patients' perioperative care according to an institute's ERAS program. Any improvements in quality of life may manifest as an earlier return to work and possibly a reduction in minor complications. Specific tools have been suggested to capture this important parameter, including (select correct answer/s):

a. Short Health Survey (SF-36)

b. American Society of Anesthesiologists (ASA) score

c. Consensus on Health Economic Criteria (CHEC) tool

d. World Health Organization Disability Assessment Schedule 2.0 (WHODAS 2.0) 